PROCEEDINGS OF THE

AMERICAN MATHEMATICAL SOCIETY

Volume 125, Number 3, March 1997, Pages 747-753

S 0002-9939(97)04005-7

\title{
CONJUGACY CLASSES OF SYMMETRIES IN ORTHOGONAL GROUPS
}

\author{
DONALD G. JAMES
}

(Communicated by Ronald M. Solomon)

\begin{abstract}
The number of conjugacy classes of symmetries in the integral orthogonal group of an indefinite $\mathbb{Z}$-lattice is determined. The results are applied to the extended Bianchi groups.
\end{abstract}

\section{INTRODUCTION}

Let $V$ be a regular quadratic space, of finite dimension $n \geq 4$, over the rational field $\mathbb{Q}$ with quadratic form $Q: V \rightarrow \mathbb{Q}$ and associated bilinear form $2 f(x, y)=$ $Q(x+y)-Q(x)-Q(y)$. Let $L$ be a $\mathbb{Z}$-lattice on $V$, let $O(L)$ be the orthogonal group of $L$, and let $O^{\prime}(L)$ be its spinor kernel. Then both $O(L)$ and $O^{\prime}(L)$ act on the symmetries

$$
\Psi(x): y \rightarrow y-2 f(x, y) Q(x)^{-1} x, \quad x, y \in L,
$$

in $O(L)$ by conjugation, with $\phi \Psi(x) \phi^{-1}=\Psi(\phi(x))$. We study the number of conjugacy classes under both actions, and then apply the results to the extended Bianchi groups and Hilbert modular groups.

The lattice $L$ represents $c \in \mathbb{Z}$ if there exists an $x \in L$ with $Q(x)=c$. The representation is primitive if $\mathbb{Z} x$ is a direct summand of $L$. Since the symmetry $\Psi(x)$ is to be integral, only primitive representations that satisfy the extra condition $2 f(x, L) \subseteq c \mathbb{Z}$ will be considered. Let $N(L, c)$ be the number of these primitive representations of $c$ modulo the action of $O(L)$. Then $N(L, c)$ also counts the number of conjugacy classes of symmetries $\Psi(x)$ with $x$ primitive and $Q(x)=c$. Let $N^{\prime}(L, c)$ be the number of these primitive representations of $c$ modulo the action of $O^{\prime}(L)$, and let $N^{\prime}\left(L_{p}, c\right)$ be the corresponding number of primitive representations by the local lattice $L_{p}$ over the $p$-adic integers $\mathbb{Z}_{p}$. Using Kneser's Strong Approximation Theorem to set up a bijection between the global and corresponding set of local orbits, as in the proof of Theorem 2.3 in [5], or Theorem 4.1 in [6], gives the following product formula.

Theorem 1.1. Let $L$ be a lattice on $V$ with $f(L, L) \subseteq \mathbb{Z}$ and discriminant $D$. Assume $c \neq 0$ and the Witt index $i_{\infty}(V \perp\langle-c\rangle) \geq 2$. Then, for $n \geq 4$,

$$
N^{\prime}(L, c)=\prod_{p \mid 2 D} N^{\prime}\left(L_{p}, c\right)<\infty .
$$

Received by the editors October 18, 1995.

1991 Mathematics Subject Classification. Primary 11E57, 11F06, 20 G30.

The author was supported by NSA grant MDA904-94-H-2034 and NSF grant DMS-95-00533.

(C)1997 American Mathematical Society 
The number of conjugacy classes of symmetries under the action of $O^{\prime}(L)$ is then determined from the action of $-I$ on the $O^{\prime}(L)$-orbits. The value $N(L, c)$ can be studied via the action of the quotient group $O(L) / O^{\prime}(L)$ on the $O^{\prime}(L)$ orbits. These methods will be used to study the conjugacy classes of symmetries in $O(L)$ in a special case corresponding to the Bianchi groups and Hilbert modular groups $P S L\left(2, \mathcal{O}_{d}\right)$. Related methods were used in [6] to classify the maximal nonelementary Fuchsian subgroups of the Bianchi groups up to conjugacy. In [11], Vulakh studied the conjugacy classes of reflections in the extended Bianchi group $R B_{d}$. Via the identification of $R B_{d}$ with a suitable group $O(L)$ (see $[2, \S 11]$ or $[10])$, this is essentially the same as studying the conjugacy classes of symmetries for this $O(L)$. Theorem 3.5 is a generalization of one of Vulakh's results. Theorem 3.6 gives an analogue for the conjugacy classes in $R B_{d}$ under the action of the Bianchi group. A Hilbert modular group analogue is simultaneously established. See also $[2, \S 11]$ for an earlier special case obtained using Siegel's analytic theory of indefinite forms.

The next section contains some general results evaluating $N^{\prime}\left(L_{p}, c\right)$. They can easily be modified to give a function field analogue where $\mathbb{Z}$ is replaced by the polynomial ring $\mathbb{F}[X]($ with $2 \neq 0)$.

\section{LOCAL SPINOR ORBITS}

Local isometry invariants on $x \in L_{p}$ under the action of $O\left(L_{p}\right)$ are given in [7] and [9]. In general they are complicated; however, for odd $p$, the restriction $f\left(x, L_{p}\right) \subseteq Q(x) \mathbb{Z}_{p}$ means that $\mathbb{Z}_{p} x$ splits $L_{p}$ as a rank one orthogonal summand (see $[8, \S 92: 6])$. For each prime $p$ let

$$
L_{p}=J_{1} \perp \cdots \perp J_{t}
$$

be a Jordan splitting of $L_{p}$ (see $[8, \S 91 \mathrm{C}]$ ), where each $J_{i}$ is a $p^{r_{i}}$-modular lattice, $r_{1}<\cdots<r_{t}$, and $n_{i}=\operatorname{rank} J_{i}$ are invariants of $L_{p}$. For odd $p$ the discriminants $d_{i}=\left(\operatorname{det} J_{i}\right) \mathfrak{u}_{p}^{2}$, where $\mathfrak{u}_{p}$ denotes the $p$-adic units, are also invariants. Note, for $p$ odd, $\left[S O\left(L_{p}\right): O^{\prime}\left(L_{p}\right)\right]=4$ whenever some $n_{i} \geq 2$, and the $r_{i}$ are not all even or not all odd (see $[8, \S 92: 5])$. When all $r_{i}$ have the same parity, $\left[S O\left(L_{p}\right): O^{\prime}\left(L_{p}\right)\right] \leq 2$. Also, $S O\left(L_{p}\right)=O^{\prime}\left(L_{p}\right)$ if and only if all $n_{i}=1$, and all the square classes $d_{i} \mathbb{Q}_{p}^{* 2}$ are the same (in particular, the $r_{i}$ all have the same parity).

If $\Psi(x) \in O(L)$ with $x$ primitive and $Q(x)=c$, then $\mathbb{Z}_{p} x$ splits $L_{p}$ as an orthogonal summand for each odd $p$. In particular, this implies that a Jordan splitting can be chosen with $\mathbb{Z}_{p} x \subseteq J_{i}$ for some $i$. Hence $c p^{-r_{i}} \in \mathfrak{u}_{p}$, and this is therefore a necessary condition for $N\left(L_{p}, c\right)>0$.

Theorem 2.1. Let $p$ be odd. Assume $c p^{-r_{i}} \in \mathfrak{u}_{p}$ for some $i$. Then

1. $N^{\prime}\left(L_{p}, c\right)=0$ if $n_{i}=1$ and $\left(\frac{c / d_{i}}{p}\right)=-1$.

2. $N^{\prime}\left(L_{p}, c\right)=2$ if $n_{i}=1,\left(\frac{c / d_{i}}{p}\right)=1$, and $r_{j}-r_{i}$ is odd for $j \neq i$.

3. $N^{\prime}\left(L_{p}, c\right)=2$ if all $n_{j}=1,\left(\frac{c / d_{i}}{p}\right)=1$, and the $d_{j} \mathbb{Q}_{p}^{* 2}$, for $j \neq i$, take at most two values, and these values are not $c \mathbb{Q}_{p}^{* 2}$.

4. $N^{\prime}\left(L_{p}, c\right)=2$ if $n_{i}=2$, all other $n_{j}=1$, and the $d_{j} \mathbb{Q}_{p}^{* 2}$, for $j \neq i$, and $d_{i} c^{-1} \mathbb{Q}_{p}^{* 2}$ take at most two values, and the product of any two values is not $\epsilon \mathbb{Q}_{p}^{* 2}$ with $\epsilon$ a non-square unit.

5. $N^{\prime}\left(L_{p}, c\right)=1$ otherwise. 
Proof. Assume first $n_{i}=1$. Let $x \in L_{p}$ be primitive with $Q(x)=c$. Then we may choose $J_{i}=\mathbb{Z}_{p} x$ and hence $c \in d_{i} \mathfrak{u}_{p}^{2}$. In this case, the representations of $c$ by $x$ and $-x$ are not spinor equivalent when $r_{j}-r_{i}$ is odd for all $j \neq i$. For assume $\phi \in O^{\prime}\left(L_{p}\right)$ with $\phi(x)=-x$. Then $\Psi(x) \phi$ fixes $x$, and hence can be viewed as an isometry on the orthogonal complement of $J_{i}$. Therefore, by [8, §92:4], $\Psi(x) \phi$ is a product of an odd number of symmetries $\Psi(y)$ with each $Q(y) p^{-r_{j}}$ a unit for some $j$. Calculating spinor norms then gives a contradiction. A similar argument holds if all $n_{j}=1$ as in case 3 . Any two representations are $S O\left(L_{p}\right)$-equivalent (see [7]), and it follows that $N^{\prime}\left(L_{p}, c\right) \leq 2$ if some $n_{j} \geq 2$, since the orthogonal complement of $x$ then admits isometries with all spinor norms in $\mathfrak{u}_{p} \mathbb{Q}_{p}^{* 2}$. When all $n_{j}=1$, it still follows that $N^{\prime}\left(L_{p}, c\right) \leq 2$, because if all the $d_{j} \mathbb{Q}_{p}^{* 2}, j \neq i$, are the same, then $\left[S O\left(L_{p}\right): O^{\prime}\left(L_{p}\right)\right] \leq 2$. In case $4, x$ and $\theta(x)$ are not spinor equivalent for $\theta \in S O\left(J_{i}\right)$ with spinor norm $\epsilon \mathbb{Q}_{p}^{* 2}$; take $\theta(x)=-x$ if $d_{i} \notin \mathbb{Q}_{p}^{* 2}$.

Now consider the case $n_{i} \geq 3$, or $n_{i}=2$ and $n_{j} \geq 2$ for some $j \neq i$. If $x$ and $y$ both represent $c$, by [7] there exists $\phi \in S O\left(L_{p}\right)$ with $\phi(x)=y$. It is easy to adjust and get $\phi \in O^{\prime}\left(L_{p}\right)$ using the orthogonal complement of $x$. The remaining cases are similar.

Corollary 2.2. The two spinor orbits in case 4 with $d_{i} \notin \mathbb{Q}_{p}^{* 2}, 2$ or 3 , are interchanged by $-I$. The two spinor orbits in case 2 are interchanged by any $\phi \in S O\left(L_{p}\right)$ with spinor norm $\eta p \mathbb{Q}_{p}^{* 2}, \eta$ a unit, and are fixed by all $\psi \in S O\left(L_{p}\right)$ with spinor norm $\eta \mathbb{Q}_{p}^{* 2}$.

Proof. Assume $Q( \pm x)=c$. There exists $\theta \in O\left(L_{p}\right)$ fixing $x$ and with $\phi \theta \Psi(x) \in$ $O^{\prime}\left(L_{p}\right)$, using the orthogonal complement of $x$. Hence $x$ is spinor equivalent to $\phi(-x)$. For the remaining part choose $\theta \in S O\left(L_{p}\right)$ fixing $x$ and with $\psi \theta \in O^{\prime}\left(L_{p}\right)$. Then $x$ is spinor equivalent to $\psi(x)$.

A dyadic unimodular lattice $L_{2}$ is even when $Q\left(L_{2}\right) \subseteq 2 \mathbb{Z}_{2}$. Otherwise $L_{2}$ is odd and has an orthogonal basis $e_{1}, \ldots, e_{n}$. Then $x=\sum_{i} a_{i} e_{i} \in L_{2}$ is characteristic if all coefficients $a_{i}$ are units (see [5], [9]).

Theorem 2.3. Let $L_{2}$ be a dyadic unimodular lattice. Then

1. $N^{\prime}\left(L_{2}, c\right)=0$ when $c \in 4 \mathbb{Z}_{2}$, or $L_{2}$ is even with $c$ a unit.

2. $N^{\prime}\left(L_{2}, c\right)=3$ when $L_{2}$ is odd and $c \equiv \sum_{i} Q\left(e_{i}\right) \not \equiv 0,4 \bmod 8$.

3. $N^{\prime}\left(L_{2}, c\right)=1$ otherwise.

Proof. Let $x \in L_{2}$ be primitive with $Q(x)=c$. Then $2 f\left(x, L_{2}\right) \subseteq c \mathbb{Z}_{2}$ forces $c \mid 2$. When $L_{2}$ is even, it is split by a hyperbolic plane $\mathbb{Z}_{2} u+\mathbb{Z}_{2} v$, and $x$ is spinor equivalent to $u+2^{-1} c v$ (see [5] or [9, $\left.\S 2\right]$ ). Now assume that $L_{2}$ is odd and $x=$ $\sum_{i} a_{i} e_{i}$ is characteristic. Then $c=Q(x)=\sum_{i} a_{i}^{2} Q\left(e_{i}\right) \equiv \sum_{i} Q\left(e_{i}\right) \bmod 8$. By Hensel's lemma, this is a necessary and sufficient condition for the existence of a characteristic representation of $c$. When $c \equiv 0 \bmod 4$ the restriction $c \mid 2$ is violated. The result follows by strengthening the arguments in Lemmas 4.3 and 5.7 in [5], or as in Theorem 2.1 above using $[9, \S 2]$.

Corollary 2.4. When $N^{\prime}\left(L_{2}, c\right)=3$, the two orbits corresponding to the characteristic representations are interchanged by $-I$ if $n$ is odd, but are fixed by $-I$ if $n$ is even.

Proof. The action of each $\Psi\left(e_{i}\right)$ interchanges the two characteristic orbits (see Theorem 2.2 in [5]). 
For the final two theorems in this section we assume, as in Theorem 1.1, that $L$ is a $\mathbb{Z}$-lattice with $f(L, L) \subseteq \mathbb{Z}$ and $n \geq 4$, that $c \neq 0$ and the index $i_{\infty}(V \perp\langle-c\rangle) \geq 2$. Also assume $L_{2}$ is unimodular.

Theorem 2.5. Necessary and sufficient conditions for $N(L, c)>0$ are:

1. $c \not \equiv 0 \bmod 4$, and $2 \mid c$ when $L_{2}$ is even,

2. at each odd prime, $c p^{-r_{i}} \in \mathfrak{u}_{p}$ for some $i$, and moreover, if $n_{i}=1$ then $\left(\frac{c / d_{i}}{p}\right)=1$.

By Theorem 1 in [4], it suffices to assume $V$ indefinite in Theorem 2.5 (instead of $\left.i_{\infty}(V \perp\langle-c\rangle) \geq 2\right)$.

Let $m$ be the number of odd primes $p$ where $N^{\prime}\left(L_{p}, c\right)=2$.

Theorem 2.6. Assume $N(L, c)>0$. When $m \geq 1$ and not only case 4 with $d_{i} \in$ $\mathbb{Q}_{p}^{* 2}$ occurs, the number of conjugacy classes of symmetries $\Psi(x)$ with $x$ primitive and $Q(x)=c$ under the action of $O^{\prime}(L)$ is $2^{m-1} N^{\prime}\left(L_{2}, c\right)$. If $m=0$ and $N^{\prime}\left(L_{2}, c\right)=$ 3 , the number of conjugacy classes is 2 when $n$ is odd, and 3 when $n$ is even.

Proof. When $m \geq 1$, this follows since $\Psi(x)=\Psi(-x)$, but $x$ and $-x$ are in different $O^{\prime}(L)$-orbits by Corollary 2.2. Use 2.4 when $m=0$.

The result is different if $m \geq 1$ and only case 4 with $d_{i} \in \mathbb{Q}_{p}^{* 2}$ occurs, since $-I$ now fixes all local $p$-adic orbits for $p$ odd.

\section{Extended BiAnCHI GROUpS}

Let $\mathcal{O}_{d}$ be the ring of integers in $\mathbb{Q}(\sqrt{ } d)$, where $d$ is a square-free integer. It was shown in [6] that the Bianchi group $P S L\left(2, \mathcal{O}_{d}\right)$, for $d<0$ and $d \equiv 2,3 \bmod 4$, is isomorphic to $O^{\prime}(L)$, where

$$
L=\mathbb{Z} r \perp \mathbb{Z} s \perp(\mathbb{Z} u+\mathbb{Z} v)=B \perp H
$$

is the lattice with $Q(r)=2, Q(s)=-2 d$, and $u, v$ are isotropic with $f(u, v)=$ $d$. The extended Bianchi group $B_{d}$, namely, the maximal discrete extension of $P S L\left(2, \mathcal{O}_{d}\right)$ in $P S L(2, \mathbb{C})$, is isomorphic to $P S O(L)$. The extended Bianchi group $R B_{d}$ is $B_{d}$ with the action of complex conjugation adjoined; it is isomorphic to $P O(L)$. The Hilbert modular group, where $d>0$, is also isomorphic to $O^{\prime}(L)$. For $d \equiv 1 \bmod 4, L$ must be replaced by $M=L+\mathbb{Z} 2^{-1}(r-s)$. These isomorphisms are related to those constructed over commutative rings in [3, $\S 7.3 \mathrm{~B}]$.

Let

$$
J=\{x \in L \mid Q(x) \in 2 d \mathbb{Z}\}=\mathbb{Z} d r \perp \mathbb{Z} s \perp H
$$

and

$$
K=\{x \in L \mid f(x, J) \subseteq 2 d \mathbb{Z}\}=\mathbb{Z} r \perp \mathbb{Z} s \perp 2 H .
$$

Then $J$ and $K$ are sublattices of $L$ that are invariant under the action of $O(L)$. Note that $O(J)=O(L)$. Clearly $O(L) \subseteq O(J)$. Conversely, let $\phi \in O(J)$ with $\phi(r) \in V$. Since $f(\phi(r), J)=f(r, J)=2 d \mathbb{Z}$, it follows that $\phi(r) \in K$ and $\phi \in O(L)$. Therefore, scaling $J$ by $d^{-1}, O(L)$ is isomorphic to the orthogonal group of the integral form $d X_{1}^{2}-X_{2}^{2}+X_{3} X_{4}$ used in [2].

We now study the conjugacy classes of symmetries in $O(L)$ and $O(M)$. For the symmetry $\Psi(x)$, with $x$ primitive in $L$ and $Q(x)=2 c$, to be integral we need $f(x, L) \subseteq c \mathbb{Z}$, and hence $Q(x)$ divides $4 d$. First determine $N^{\prime}(L, 2 c)=$ $\prod_{p \mid 2 d} N^{\prime}\left(L_{p}, 2 c\right)$ for each $Q(x)=2 c \mid 4 d$. Then $N(L, 2 c)$ is obtained from the action 
of the quotient group $O(L) / O^{\prime}(L)$ on the $O^{\prime}(L)$-orbits. From [6], $\left[O(L): O^{\prime}(L)\right]=$ $2^{t+2}$ where $t$ is the number of distinct prime divisors of the discriminant of $\mathbb{Q}(\sqrt{ } d)$.

For $w \in \mathbb{Z} r \perp \mathbb{Z} d^{-1} s$ and $x \in L$, let

$$
E(u, w)(x)=x-f(u, x) w+f(w, x) u-2^{-1} Q(w) f(u, x) u .
$$

The Eichler transformation $E(u, w)$ lies in $O^{\prime}(L)$.

Theorem 3.1. Let $d$ be even. Then

1. $N^{\prime}\left(L_{2}, 2 c\right)=0$ for $c \equiv 0 \bmod 4$.

2. $N^{\prime}\left(L_{2}, 2 c\right)=1$ for $c \equiv 1+d,-3 \bmod 8$.

3. $N^{\prime}\left(L_{2}, 2 c\right)=2$ for $c \equiv \pm 2 \bmod 8$.

4. $N^{\prime}\left(L_{2}, 2 c\right)=3$ for $c \equiv 1,1-d \bmod 8$.

Proof. Let $x=a_{1} r+a_{2} s+b u+b^{\prime} v \in L_{2}$ with $Q(x)=2 c$. When $4 \mid c$ the condition $f\left(x, L_{2}\right) \subseteq 4 \mathbb{Z}_{2}$ forces $x \in 2 L_{2}$ so that $N^{\prime}\left(L_{2}, 2 c\right)=0$. Assume $c \not \equiv 0 \bmod 4$. If $x \notin K_{2}$, we may assume $b$ is a unit and then $x$ is spinor equivalent, via a suitable $E(v, w) \in O^{\prime}(L)$, to $b u+(b d)^{-1} c v$ for $c$ even, or to $r+b u+(b d)^{-1}(c-1) v$ for $c$ odd. The map $\tau$ fixing $r$ and $s$, and sending $u$ to $b^{-1} u$, has spinor norm $b \mathbb{Q}_{2}^{* 2}$. In both cases there exists $\sigma \in S O(L)$ fixing $x$ with spinor norm $b \mathbb{Q}_{2}^{* 2}$ (use Theorem 3.14 in [1] on the orthogonal complement of $x$ when $c$ is odd). Then $\tau \sigma \in O^{\prime}\left(L_{2}\right)$, so we may assume $b=1$. Thus, up to spinor equivalence, there is only one representation $x \notin K_{2}$ with $Q(x)=2 c$.

If $x \in K_{2}$, then $c \equiv a_{1}^{2}-d a_{2}^{2} \equiv 1,1-d, \pm 2 \bmod 8$. Then, by modifying the previous argument, get $b=2$. When $c$ is even, a suitable $E(v, w)$ sends $x$ to a uniquely determined $a r+s+2 u+2 b^{\prime} v$ with $a=0$ or 2 . When $c$ is odd, $x$ can be sent to $\pm r+a s+2 u+2 b^{\prime} v$, with $a=0$ or 1 unique for $x$. The two sign choices lie in different spinor orbits by Theorem 3.14(i) in [1], since the orthogonal complement of $x$ only allows isometries with spinor norm a unit.

Corollary 3.2. $N\left(L_{2}, 2 c\right)=2$ when $c \equiv 1,1-d, \pm 2 \bmod 8$.

Proof. The $x \in K_{2}$ are analogous to the characteristic $x$ in the dyadic unimodular case. They determine an orbit that cannot be interchanged by $O\left(L_{2}\right)$ with an orbit given by an $x \notin K_{2}$. The two characteristic orbits are interchanged by $-I$ when $c \equiv 1,1-d \bmod 8$.

Theorem 3.3. Let $d \equiv 3 \bmod 4$. Then

1. $N^{\prime}\left(L_{2}, 2 c\right)=0$ for $c \equiv 0 \bmod 4$.

2. $N^{\prime}\left(L_{2}, 2 c\right)=1$ for $c \equiv 2,3 \bmod 4$.

3. $N^{\prime}\left(L_{2}, 2 c\right)=3$ for $c \equiv 1 \bmod 4$.

Proof. Let $x=a_{1} r+a_{2} s+b_{1} u+b_{2} v \in L_{2}$ be primitive with $Q(x)=2 c$ and $f\left(x, L_{2}\right) \subseteq$ $c \mathbb{Z}_{2}$. Then $c|2, c| b_{1}$ and $c \mid b_{2}$. Hence there are no solutions when $c \equiv 0 \bmod 4$, and $x$ is spinor equivalent to $r+s+2 u+2 b v \in K_{2}$ when $c \equiv 2 \bmod 4$. Now assume $c$ is a unit. Then $u+c d^{-1} v$ is a representation of $2 c$, and $r+2 u+(2 d)^{-1}(c-1) v$ and $s+2 u+(2 d)^{-1}(c+d) v \in K_{2}$ give spinor inequivalent representations when $c \equiv 1$ mod 4 . These are distinct by [1], and are the only ones by arguments similar to those in Theorem 3.1 .

Corollary 3.4. $N\left(L_{2}, 2 c\right)=2$ when $c \equiv 1 \bmod 4$.

Proof. The two orbits corresponding to the $x \in K_{2}$ are interchanged by $\Psi(r-s) \in$ $O\left(L_{2}\right)$. 
For $p$ a prime dividing $d$, put $d=p q$ and choose $a, b \in \mathbb{Z}$ with $a q+b p=1$. Then $\sigma(p)=-\Psi(u+b v) \Psi(b p r+a u+b v) \in S O(L)$ has spinor norm $p \mathbb{Q}^{* 2}$. Let $m=m(c)$ be the number of odd primes dividing $d$ with $(p, c)=1$ and $\left(\frac{c}{p}\right)=1$. The following gives the number $N(L, c)$ of conjugacy classes under $O(L)$ of symmetries $\Psi(x)$ with $x$ primitive and $Q(x)=2 c$.

Theorem 3.5. Assume that $c>0$ when $d<0$, that $N(M, 2 c)>0$ when $d \equiv 1$ $\bmod 4$, and $N(L, 2 c)>0$ when $d \equiv 2,3 \bmod 4$. Then

1. $N(M, 2 c)=2^{-m} N^{\prime}(M, 2 c)=1$ for $d \equiv 1 \bmod 4$.

2. $N(L, 2 c)=2^{-m} N^{\prime}(L, 2 c)=N^{\prime}\left(L_{2}, 2 c\right)$ for $c \not \equiv 1,1-d$ mod 8 when $d$ is even.

3. $N(L, 2 c)=1$ and $N^{\prime}(L, 2 c)=2^{m}$ for $c \equiv 2,3 \bmod 4$ when $d \equiv 3 \bmod 4$.

4. $N(L, 2 c)=2$ and $N^{\prime}(L, 2 c)=2^{m} 3$ for $c \equiv-d \equiv 1 \bmod 4$, and for $c \equiv 1,1-d$ mod 8 when $d$ is even.

Proof. When $d \equiv 1 \bmod 4, M_{2}$ is an even unimodular lattice and hence, by Theorems 1.1 and 2.1, $N^{\prime}(M, 2 c)=2^{m}$. The group $O(M)$ is generated over $O^{\prime}(M)$ by $\sigma\left(p_{i}\right), 1 \leq i \leq t, \Psi(u-v)$ and $\Psi(u+v)$. The result now follows since $\sigma\left(p_{i}\right)$ with $\left(p_{i}, c\right)=1$ interchanges the two $O^{\prime}\left(M_{p_{i}}\right)$-orbits and leaves all other $O^{\prime}\left(M_{p_{j}}\right)$-orbits unchanged by Corollary 2.2. Each $\sigma(p)$ corresponding to a $p$ counted in $m$ thus independently halves the total number of $O^{\prime}(M)$-orbits. Thus $N(M, 2 c)=1$.

The argument for $d$ even is essentially the same, with $\sigma(p)$ having no effect on the local dyadic orbits for odd $p$. By Corollary 3.2 the $O^{\prime}\left(L_{2}\right)$-orbits are only effected by $O(L)$ when $c \equiv 1,1-d \bmod 8$, and then $-I$ reduces the number of dyadic orbits from 3 to 2 .

For $d \equiv 3 \bmod 4$ the dyadic orbits must again be considered when $c \equiv 1 \bmod 4$. Now

$$
\Psi(a d r+a s-2 a b u-2 v) \Psi(v-a u) \in S O(L),
$$

where $2 q=1-d$ and $a q-2 b=1$, has spinor norm $2 \mathbb{Q}^{* 2}$, interchanges the two dyadic orbits corresponding to the representatives from $K_{2}$, and fixes the local orbits at odd primes by Corollary 2.2.

This is essentially a refined version of Theorem 11 in [11] where only an upper bound is given for the total number of conjugacy classes of reflections in $R B_{d}$. Explicit examples, similar to those given in Theorem 11.3 of [2] and Theorem 12 of [11], can be constructed from the local information. That $N(M, 2 c)=1$ also follows from Theorems 2 and 4 in [4].

Theorem 3.6. Assume $d \equiv 2,3 \bmod 4$, that $c>0$ when $d<0$, and $N(L, 2 c)>0$. When $m \geq 1$, the number of conjugacy classes of symmetries $\Psi(x)$ with $x$ primitive and $Q(x)=2 c \mid 4 d$, under the action of $O^{\prime}(L)$, is $2^{m-1} N^{\prime}\left(L_{2}, 2 c\right)$. When $m=0$, this number is $N\left(L_{2}, 2 c\right)$, except for $c \equiv-d \equiv 1 \bmod 4$ where it is 3 .

Proof. The action of $-I$ halves the number of orbits by interchanging in pairs the local $O^{\prime}(L)$-orbits at odd primes when $m>0$. Use Corollary 3.2 when $m=0$.

This essentially gives the number of conjugacy classes of reflections in $R B_{d}$ under the action of $P S L\left(2, O_{d}\right), d<0$. The corresponding result for $O(M), d \equiv 1 \bmod 4$, is already covered in Theorem 2.6. 


\section{REFERENCES}

[1] A.G. Earnest and J.S. Hsia, Spinor norms of local integral rotations II, Pacific J. Math. 61 (1975), 71-86. MR 85m:11022

[2] J. Elstrodt, F. Grunewald and J. Mennicke, Discontinuous groups on three-dimensional hyperbolic space: analytical theory and arithmetic applications, Russian Math. Surveys 38 (1983), 137-168. MR 85g:11045

[3] A.J. Hahn and O.T. O'Meara, The Classical Groups and K-Theory, Springer-Verlag (1989). MR 90i:20002

[4] D.G. James, Representations by integral quadratic forms, J. Number Theory 4 (1972), 321329. MR 46:8976

[5] D.G. James, Integral sums of squares in algebraic number fields, Amer. J. Math. 113 (1991), 129-146. MR 92j:11036

[6] D.G. James and C. Maclachlan, Fuchsian subgroups of Bianchi groups, Trans. Amer. Math. Soc. 348 (1996), 1989-2002. CMP 96:09

[7] D.G. James and S.M. Rosenzweig, Associated vectors in lattices over valuation rings, Amer. J. Math. 90 (1968), 295-307. MR 36:3754

[8] O.T. O'Meara, Introduction to Quadratic Forms, Springer-Verlag (1963). MR 27:2485

[9] A. Trojan, The integral extension of isometries of quadratic forms over local fields, Canadian J. Math. 18 (1966), 920-942. MR 34:2561

[10] E.B. Vinberg, Reflective subgroups in Bianchi groups, Selecta Math. Sov. 9 (1990), 309-314. MR 91j:20117

[11] L.Va. Vulakh, Reflections in extended Bianchi groups, Math. Proc. Camb. Phil. Soc. 115 (1994), 13-25. MR 94k:20086

Department of Mathematics, Pennsylvania State University, University Park, PennSYLVANIA 16802

E-mail address: james@math.psu.edu 